\title{
CASE STUDY OF THE OPTIMIZING THE AUTOMOTIVE MANUFACTURING SYSTEMS EFFICIENCY VIA APPLYING NEW METHOD OF SCHEDULING
}

\author{
Seyedehfarzaneh Nojabaei and Matthew Franchetti \\ Mechanical, Industrial and Manufacturing Engineering (MIME), \\ The University of Toledo, Toledo, OH 43606 USA
}

Received 2013-10-15; Revised 2014-06-30; Accepted 2014-07-07

\begin{abstract}
Efficiency is becoming a pivotal aspect in each manufacturing system and scheduling plays a crucial role in sustaining it. The applicability of distributed computing to coordinate and execute jobs has been investigated in the past literature. Moreover, it is significant that even for sensitive industrial systems the only criterion of allocating jobs to appropriate machines is the FIFO policy. On the other flip, many researchers are of the opinion that the main reason behind failing to provide fairness in distributed systems is considering the only criterion of time stamp to judge upon and form the queue of jobs with the aim of allocating those jobs to the machines. In order to increase the efficiency of sensitive industrial system, this study takes into consideration of three criteria of each job including priority, time action and time stamp. The methodology adopted by this study is definition of job scheduler and positioning jobs in temporary queue and sorting via developing bubble sort. In sorting algorithm criterion of priority, time action should be considered besides time stamp to recognize the tense jobs for processing earlier. To evaluate this algorithm first a numerical test case (simulation) is programmed and then the case study performing in order to optimize efficiency of applying this method in real manufacturing system. Eventually the results of this study provided evidence on that the rate of efficiency is increased.
\end{abstract}

Keywords: Scheduling, Efficiency, Automotive Manufacturing Systems, Distributed Control System, Optimization

\section{INTRODUCTION}

Scheduling includes clarifying plan and priority of the jobs that should be performed in a predetermined operation. It means that within an organization, scheduling pertains to setting the timing of performing particular resources in the system. So, it is directly related to the use of equipment, facilities and human activities and it maximizes capacity utilization and system efficiency. Scheduling is practiced in almost every organization regardless of the nature of its activities and appropriate scheduling makes efficients use of the capacity (Taiwo, 2007). It means that manufacturers should schedule production via developing schedule for labors, equipments, acquisitions, design, manufacturing, industrialization, maintenance and even for after-sale services. Even though, a wide discrepancy and disagreement exist between the scholars on this issue. The scope of scheduling has wide horizon and the state-of-the-art dimensions of which have been concealed. Scheduling problems cope with the allocation of resources (material, labor, technology) to a set of activities during a period of time (Baker, 1974). The scheduling must be designed in a way to keep processors

Corresponding Author: Seyedehfarzaneh Nojabaei, Mechanical, Industrial and Manufacturing Engineering (MIME), The University of Toledo, Toledo, $\mathrm{OH} 43606$ USA 
busy by efficiently distributing the workload, usually in terms of response time, resource availability and maximum throughput of application (Samreen and Khiyal, 2007). Emami-Mehrgani et al. (2011) discussed that industry strives to cut down on production costs and optimize profit through operating methods consistent with various legal requirements. In this regard, numerous of scholars have made attempts to schedule via divergent methods including classical\& fuzzy logic scheduling (Tzimopoulos et al., 2008) genetic algorithm (Taghavifard et al., 2009; Gao and Liu, 2007), neural network artificial (Venkatachalam et al., 2008), fuzzy logic (Omar, 2007) and programming model (Chen, 2010), real time scheduling algorithm (Ahmad and Othman, 2010), Petri nets (Ghoul et al., 2007) and hierarchical scheduling (Omar et al., 2005) so on.

Non-traditional optimization technique such as Neural Network (NN) technique provides a complete solution methodology for solving the shop floor scheduling problems. (Venkatachalam et al., 2008).

The operations may include the crude evaluation, selection, scheduling and product logistics planning problems solved via developed programming model (Hassan et al., 2011).

Muzaffer and Alikalfa (2011) proposed that scheduling is a very indispensable task for manufacturing systems in today's harsh competitive markets and ever soaring massive number of research efforts have investigated the solution of the scheduling problems. No need to say that the scheduling problems cannot be solved optimally for even modest problem sizes. Pinedo (2012) described that scheduling problems cope with the allocation of resources (material, labor, technology) to carry out a set of activities during a period of time. Studies in manufacturing scheduling mostly deal with priority rules without any consideration to the states of the system sometimes due to ease of use in the shop floor. In traditional manufacturing systems, scheduling is carried out by machine operators and shop supervisors (Muzaffer and Alikalfa, 2011). Therefore complex, multi-attribute, or state-observing rules can cause a serious surveillance problem. Computer-Integrated Manufacturing (CIM) systems on the other hand do not face these sorts of challenges (Mahdavi and Shirazi, 2010).

A priority rule is used to determine which job from a queue is to be operated next. Numerous priority rules have been introduced in the literature. Some researchers adopted simulation and determine the most favorable priority rule (s) for the operating conditions, the production objectives and the current shop status (Chen, 2007).

The priority rules, also called dispatching or scheduling rules, have been widely used to provide good and time-efficient solutions to job-scheduling problems for decades (Pinedo, 2012). The priorityscheduling algorithm is with much more reduced waiting time for the processes (Rashid and Akhtar, 2006).

Muzaffer and Alikalfa (2011) proposed nine, most preferred priority rules for tardiness are collected from the previous studies to investigate the contribution of state-dependent priority rules. Indeed, research on effective scheduling plays crucial roles in improving production efficiency, decreasing production costs and so on. In this regard, a great number of researchers have being paid attention to this issue (Luqiao, 2010).

\subsection{Problem Statement}

According to previous works, owing to unfair decisions on job management, systems with any algorithm have crash hurdle. It seems this occurs due to unfair judgment on job incorporation into the resources or machines. If we include some of the other substantial parameters, our judgments will become fairer.

A large proportion of the published research in the field of job scheduling problem has been dedicated to allocation of $n$ jobs to $m$ machines while in the meanwhile taking scheduling with the best performance into account (Al-Hinai and Elmekkawy, 2011). On the other hand, based on scholars' viewpoints, several operations can be processed by one machine simultaneously with different priorities in our problem scope. Jobs with higher priority will be processed in short time. The control point in the proposed method is not the timing of a job being processed, but the priority of the job (Qiming et al., 2009).

Job scheduling can be viewed as an optimization problem, bounded both by the sequence and the resource constraints. In this study traditional job scheduling is considered and it is assumed that each job passes through. On account of the reviewed literature, assigning the restricted number of $n$ jobs to $m$ identical machines to assure that each job is completed before its deadline, even in the presence of faults, is defined as the problem of this investigation.

To the best knowledge of authors, there is no method of scheduling in which all the priority resources criteria, time stamp and time action simultaneously have been considered. Therefore, a more realistic scheduling model should take into account six effective criteria including machine, maintenance, process, environment, management and material activities. So in this study in order to decrease the probability of crash in manufacturing systems besides the time stamp (time of arrival) and the time action (duration time), the specific priority (The judgment is based on those six criteria 
mentioned above) of each job in terms of the amount of importance and tensing is considered.

\subsection{Objective of Paper}

According to the problem of study, a job scheduling algorithm on the principle of priority, time action and time stamp of each job is developed. Thus, first and foremost, forming temporary queue of all jobs should be considered then recognizing tense jobs based on those weight factors by considering three criteria devoted to the jobs. This algorithm should alter jobs position in the queue and provide new opportunities for tense jobs to be performed prior than the other jobs. So based on this ultimate target, the objectives of thesis are:

- Developing scheduling method with considering priority aspect in failure prone manufacturing systems

- To evaluate the performance of the proposed scheduling method in Iran Khodro Company (IKCO) case study

\section{METHODOLOGY}

Unfortunately, in the majority of car manufacturing systems the only criterion of allocating jobs to appropriate machines via schedulers of Central Control Room (CCR) is First In First Out (FIFO) policy. So the main reason of failing to provide for fairness in distributed systems, is considering the only criterion of time stamp in order to judge and form the queue of jobs with the aim of allocating those jobs to the machines. Owing to increasing the efficiency in car manufacturing system in this study three criteria of each job are being simultaneously considered. The methodology employing in this research is definition of job scheduler and positioning jobs in temporary queue and sorting via developing bubble sort in MATLAB distributed computing software, on the basis of three factors of priority, time action (duration) and time stamp. In order to evaluate proposed method first a simulation is created, besides the simulation, there is a case study in IKCO to evaluate the new method of scheduling. Eventually, by applying this evaluation we can come to this conclusion that efficiency of system is enhanced.

Figure 1 and 2 show CCR where operators can command the robots to use the suitable color.

The CCR is a place in which the schedulers (computers) are located. Hence, the function of this section plays an important role in this research. The main specifications of CCR are listed as below:

- Control room is generally defined by its design, use and location
- Control rooms allow people to control a system from a remote or centralized location

- Central control station rooms are separated from the remainder of the building

\subsection{Assumptions}

This concept considers to the manufacturing system. It is obvious that the manufacturing system, the safety of which is being threatened, is on the verge of collapse. So some preventive actions should be taken to increase fault tolerance. As below the assumptions of methodology in this study is dwelt on:

- The schedulers are distributed

- Weight factors for each job in terms of Time stamp, action time and priority should be predetermined

- The number of machine and job are restricted and infinitive

- This dissertation is restricted to the errors related to scheduling and priority of jobs, so it should be mentioned that the other errors derived from human faults are not included

- The system reliability is mixed of series system and parallel system

- The scope of this study is restricted to failure prone manufacturing or the failure prone region of industrial systems

- It should be mentioned that the queue of jobs is infinitive in issue is applicable in sorting module

- The controlling system is centralize

\subsection{Normalize Method}

Normalization refers to the division of multiple sets of data (in this study data are vector's elements) by a common variable in order to negate that variable's effect on the data. So allowing underlying characteristics of the data sets to be compared (Zang et al., 2011). This allows data on different scales to be compared, by bringing them to a common scale. In terms of levels of measurement, these ratios only make sense for ratio measurements (where ratios of measurements are meaningful), not interval measurements (where only distances are meaningful, but not ratios). Parametric normalization frequently uses pivotal quantities, so functions whose sampling distribution does not depend on the parameters and particularly ancillary statistics, pivotal quantities that can be computed from observations, without knowing parameters. 


\subsection{Sorter Methodology}

In this new algorithm of scheduling the jobs should be arranged based on three criteria of priority, time action and time stamp. As below the new algorithm of sorting by considering three criteria to arrange the jobs and determine the queue of jobs is dwelt on. Figure 3 illustrates that time action, time stamp and priority of each job should be multiple in a specific weight factor. The amount of weight factor for priority is derived from the importance of six criteria which mentioned in section 1.2 for each job. In order to devote weigh factor to the time action of each job length of time for each job can have invert role. For instance two jobs can be considered, first one is loading new program on PLC and the second one is summing of two integers with same sequence but with different time durations are received by CCR. The processing of first job takes one second and the other takes $1 / 1000$ of second. It is not fair, just a bit earlier received time caused the first job to be processed whereby CCR, it means that the other must be waited 1000 times of its time processing. So for each job by considering condition of that job the weight factor can be changed. The algorithm of sorting method in MATLAB distributed computing system is programmed.

\subsection{Evaluation of Methodology}

To evaluate of the mentioned algorithm (Nojabaei et al., 2012), simulation method for numerical test case and the case study of IKCO are dwelt on. Based on the simulated algorithm it would not be wrong when we come to this conclusion that reforming jobs in new queue eliminates the faults (errors) of systems which are derived from ignoring priority criteria aspects and time action of those jobs. Besides the numerical test case, there is a case study of applying this new scheduling in pain shop of car manufacturing system.

\subsection{Simulation Method for Numerical Test Case}

Papazachos and Karatza (2010) pointed out that distributed systems have become very popular because of significant attributes such as cost-efficiency, scalability, performance and reliability.

Parallel Computing Toolbox software and MATLAB Distributed Computing Server software enable us to coordinate and execute independent MATLAB ${ }^{\circledR}$ operations simultaneously on a cluster of computers, speeding up execution of large MATLAB jobs.

In some occasions, it is logical not to forward jobs to the machine (even in the case of the non-empty line for that machine) but to wait for the moment that the tense job will be entered the line. Such an approach may be introduced in stochastic scheduling too. A unification of both approaches seems to be more effective than each of them separately. There is no method for scheduling with considering these three criteria to enhancing the reliability of system (Nojabaei et al., 2012). The proposed method for these sensitive industrial systems can provide perfect scope in which the reliability of system will be considerably increased.

In this part, we simulate the algorithm with MATLAB DCS software. So, first we find a resource as a scheduler as shown in Fig 4 and 5:

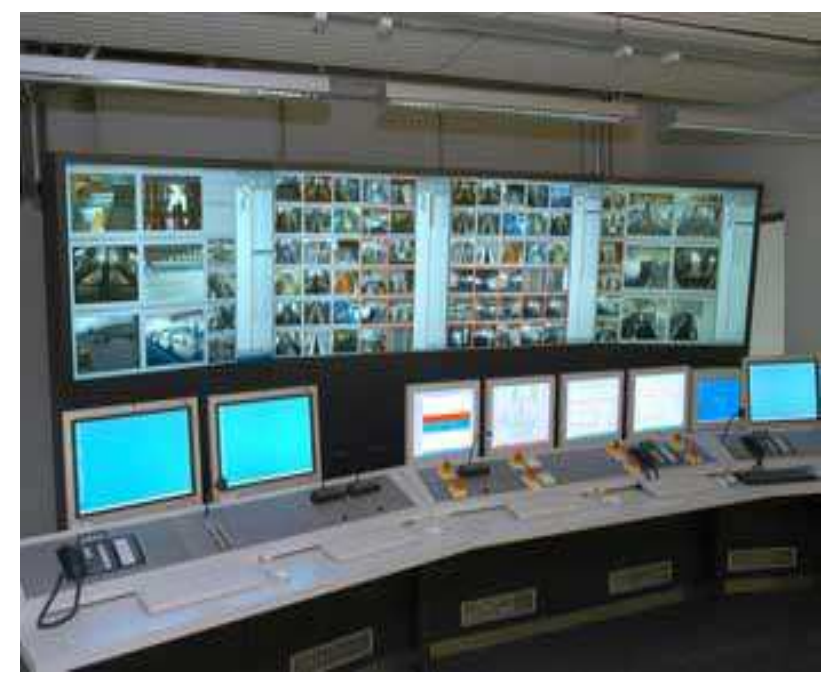

Fig. 1. Central Control Room scheduler Computers (CCR IKCO's)

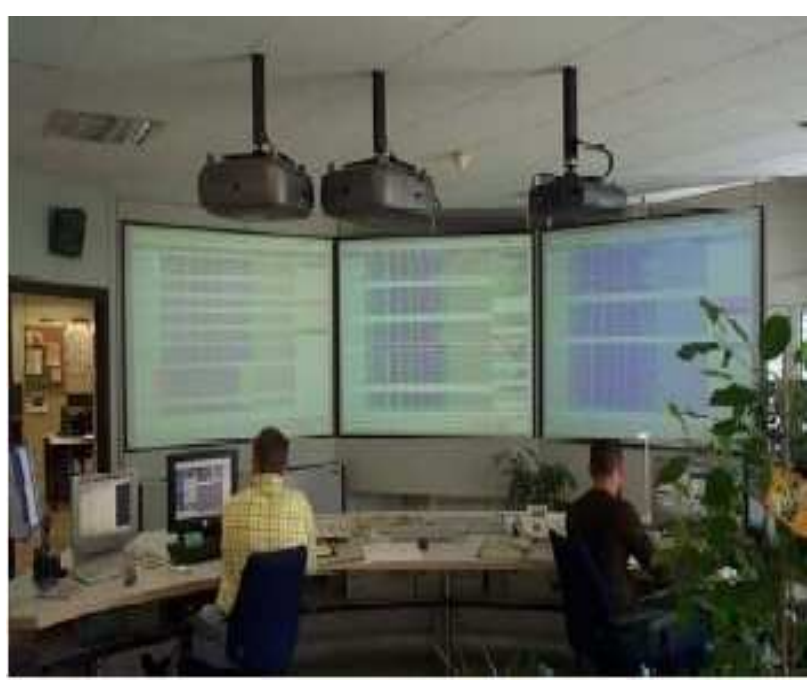

Fig. 2. Central Control Room (IKCO's CCR) 


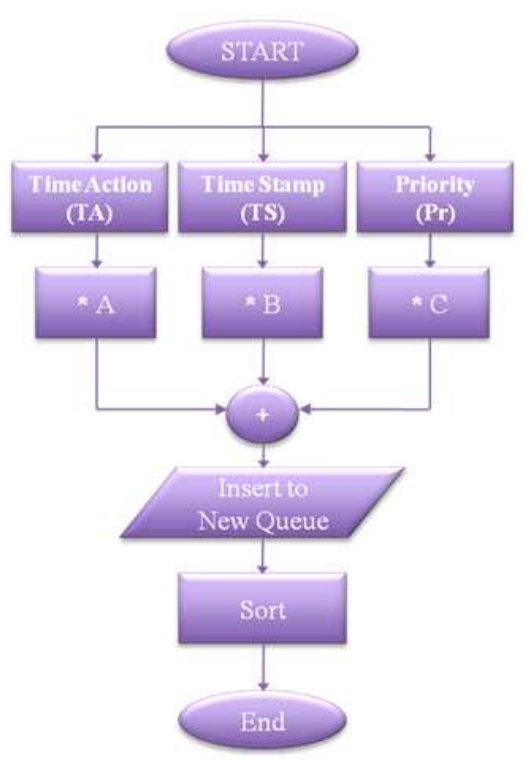

Fig. 3. Flowchart sorting algorithm

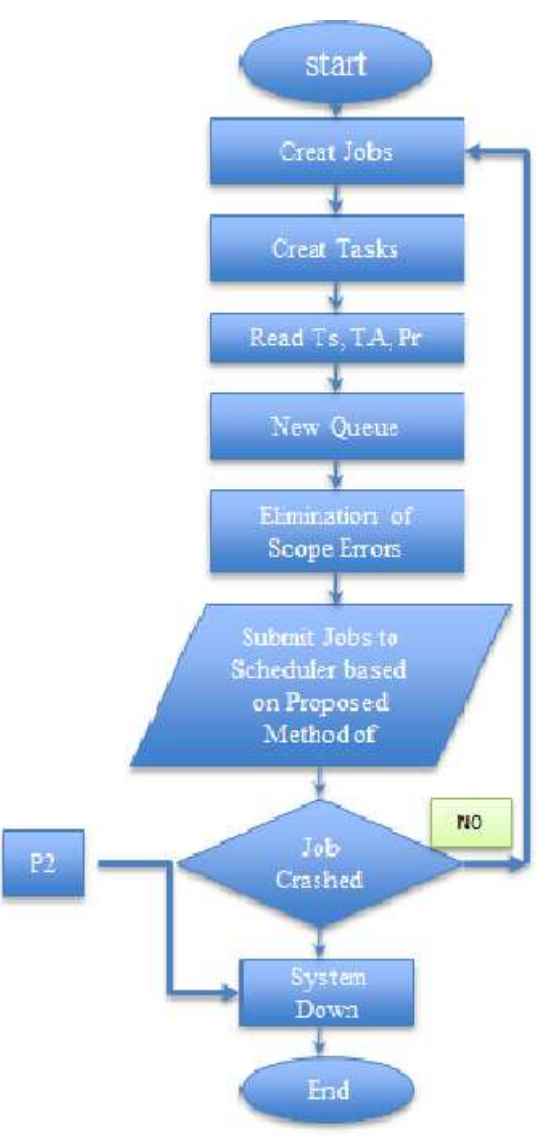

Fig. 4. The flowchart of proposed method of scheduling

Science Publications

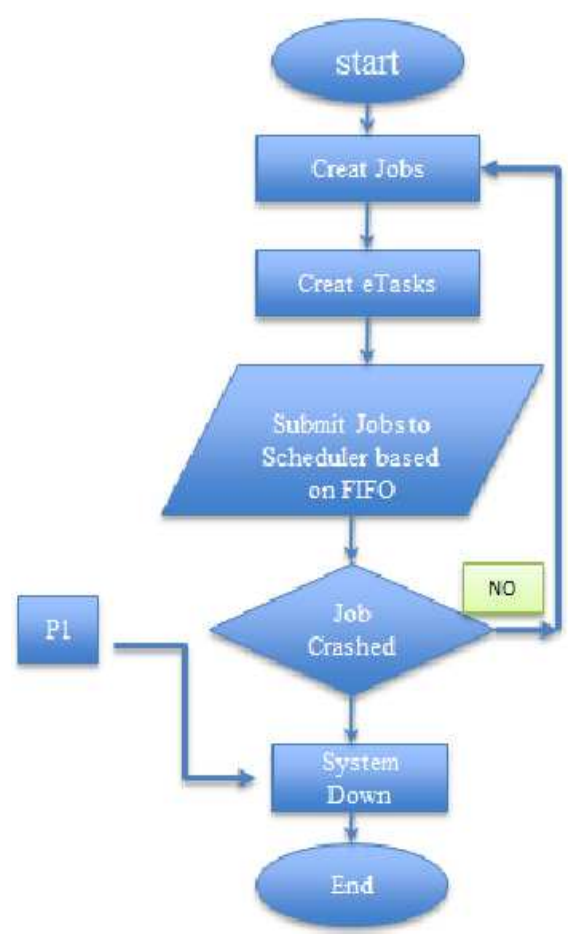

Fig. 5. The flowchart of existing method of scheduling

\subsection{Simulation Program}

Sched $=$ Find Resource ('scheduler', 'type', 'local')

Then, we create 3 jobs (job1, job2 and job3):

job1 $=$ Create Job (sched)
job2 $=$ Create Job (sched)
job3 $=$ Create Job (sched)

In third step assigns a task to each of jobs:

Create task=(job1,@sum, 1, $\left.\left\{\left[\begin{array}{ll}1 & 1\end{array}\right]\right\}\right)$

Create task=(job2,@sum, 1, $\left.\left\{\left[\begin{array}{ll}2 & 2\end{array}\right]\right\}\right)$

Create task = (job3,@sum, 1, $\left.\left\{\left[\begin{array}{ll}3 & 3\end{array}\right]\right\}\right)$

Now, we can submit all of jobs (with their tasks) to scheduler in other word, we submit jobs to the job queue. As we know, here scheduler has a coordinator effect:

$$
\begin{aligned}
& \text { Submit }=(\text { job1) } \\
& \text { Submit }=(\text { job2) } \\
& \text { Submit }=(\text { job3) }
\end{aligned}
$$

In the last step we can have the final computing with below instructions:

$\begin{array}{ll}\text { Wait for state } & =(\mathrm{j}) \\ \text { Results } & =\text { Get all output arguments }(\mathrm{j})\end{array}$ 
After this simulation, the results can be sorted with applying three important parameters (Time stamp, Time action or time duration and Priority) afterwards new queue will be reformed. By the applying the reformed queue for the system, optimized results will be obtained in result section.

\subsection{Iran Khodro Company (IKCO) Case Study}

Iran Khodro Company Also known as IKCO, is the leading Middle East automaker. IKCO produces vehicles under 11 brand names. The Iranian-designed IKCO's Samand, is Iran's "national. Plant layout of the Paint Shop in IKCO is based on arranging the processes to painting the products which is body of cars. According to various types of layouts in manufacturing design this is a Product Layout in this part of production line. In production line of this company, production is based on producing cars in almost high volume. Therefore, the production type of this company is a mass production.

\subsection{Result of Paper}

As mentioned, in the presented algorithm, After performing this simulation for several times and create random function for industrial failures as below it is revealed that applied algorithm result in better performance in terms of reducing the failure of systems. Besides, it is obvious that it will cause increase the reliability of the system via fair judgment and temporary queue. The below Figures are illustrated that the applied method results increasing performance.

Figure 6 is illustrated that the time of crashing server machine based on station and close area. As shown. It is ostensible that performance of proposed algorithm is better than previous algorithm. Subsequently, the probability of crashing will be decreased. Thus, our system is reliable and as mentioned, faults occur less than.

\subsection{Influence of New Method of Scheduling on the Efficiency of IKCO' Paint Shop}

Figure 7 depicts a comparison on efficiency of production before and after considering proposed new scheduling method in this investigation. The planned capacity of paint shop is equal to 52 automobiles units per hour. The efficiency belong to the 36 days were measured out of 52 and multiplied by 100 . Considering priority and time action of each job, beside its time stamp, in turn, causes significant enhancement of efficiency over time. On the basis of Fig. 7 all amounts of efficiencies are increased via considering the new scheduling method.

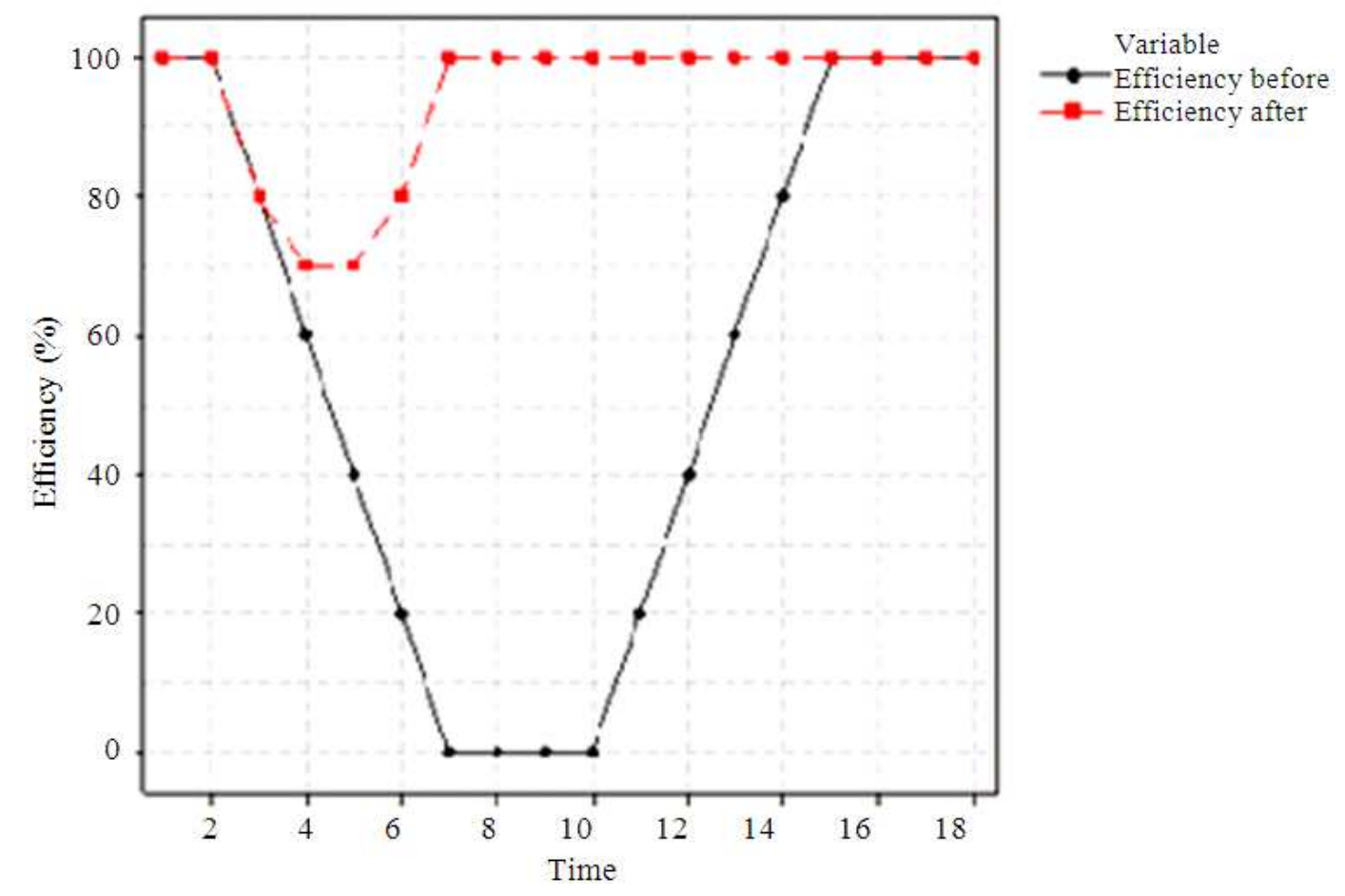

Fig. 6. Comparison of series 1 with series 2 (which can be seen from time 7 up to time 10 the system crashed) 


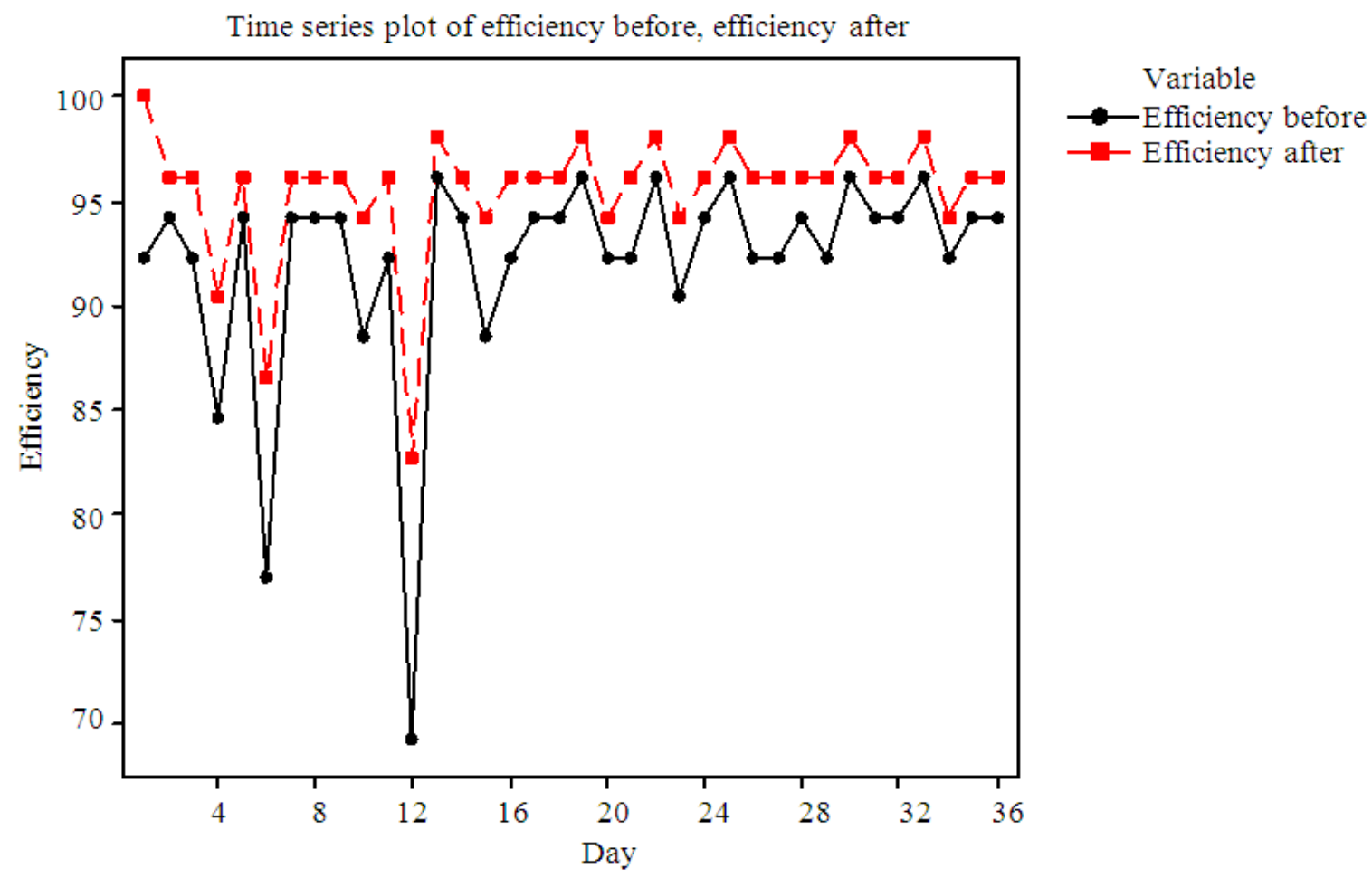

Fig. 7. Comparison of paint shop's efficiency before and after proposed scheduling

Figure 8 shows the distribution of efficiency for the 36 surveyed days of the research a mean of $92.15 \%$ is calculated. According to Fig. 8, the majority of frequencies occurred from 85 to $95 \%$ and the standard deviation of efficiency is measured by 5.34. The Fig. 8 illustrates that by average, the efficiency occurred $(-5.34$, +5.34 ) percent from mean $92.15 \%$.

Figure 8 represents the importance on efficiency while after considering new scheduling method of the research. This histogram shows that the mean of efficiency in comparison with the before status has been increased for 36 observations. The new mean efficiency is measured by 95.51 while it was calculated 92.15 before considering the new method of scheduling. Although, it is obvious that Standard Deviation appears to smaller in comparison with before status. Based on Fig. 9 Standard Deviation has improved about $2.2 \%$ it means that standard deviation decreased from 5.349 to 3.152. Therefore, according to all points which has been mentioned, it can be concluded that both mean and standard deviation improved by employing new scheduling method.
Table 1 represents a brief description based on statistics of both efficiencies before and after considering new method of scheduling. On the principle of the Table 1 number of samples are supposed to be 36 .

The variance of efficiencies for both methods are measured by 28.6 (before) and 9.932 (after). The range of observation before is about 30\% (69.231-96.154) while after employing new method of scheduling this rand is changed into (82.89-100).

Table 1 illustrates that the mean of samples for before status is 92.147 and after considering the new method of scheduling this rate has been soared to 95.513 , it means that the overall rate of production is increased over time. On the other hand, Table 1 shows that the variation scatter for after considering new scheduling method is less than before.

Figure 10 compares the approximental normal distribution of both efficiencies before and after employing the new scheduling method. Based on this histogram for 36 days as the samples the mean of efficiency soared from 92.15 to 95.51 and the standard deviation reduced from 5.349 to 3.152 . 
Seyedehfarzaneh Nojabaei and Matthew Franchetti / American Journal of Engineering and Applied Sciences 7 (2): 282-291, 2014

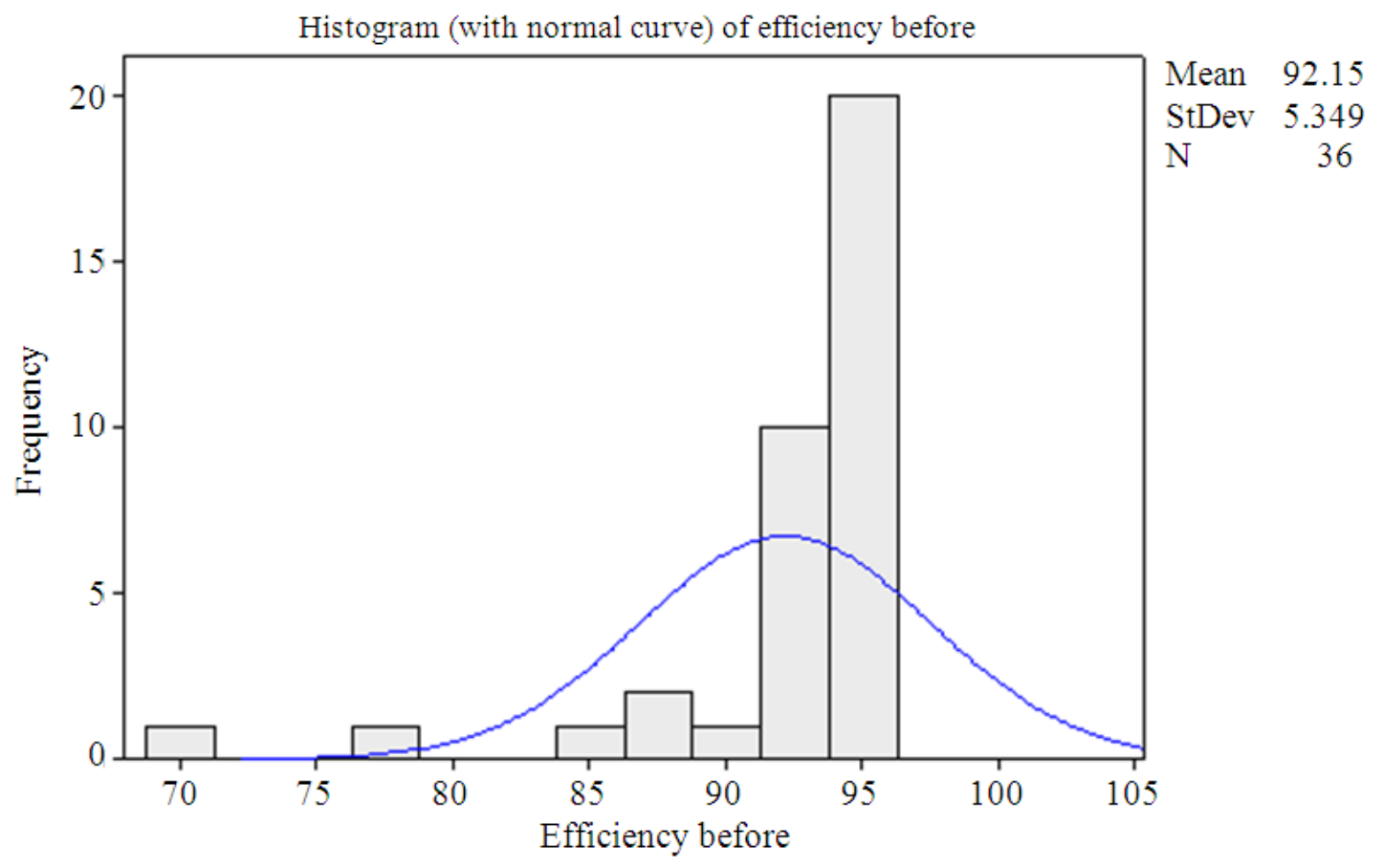

Fig. 8. Histogram of efficiency before considering new scheduling method

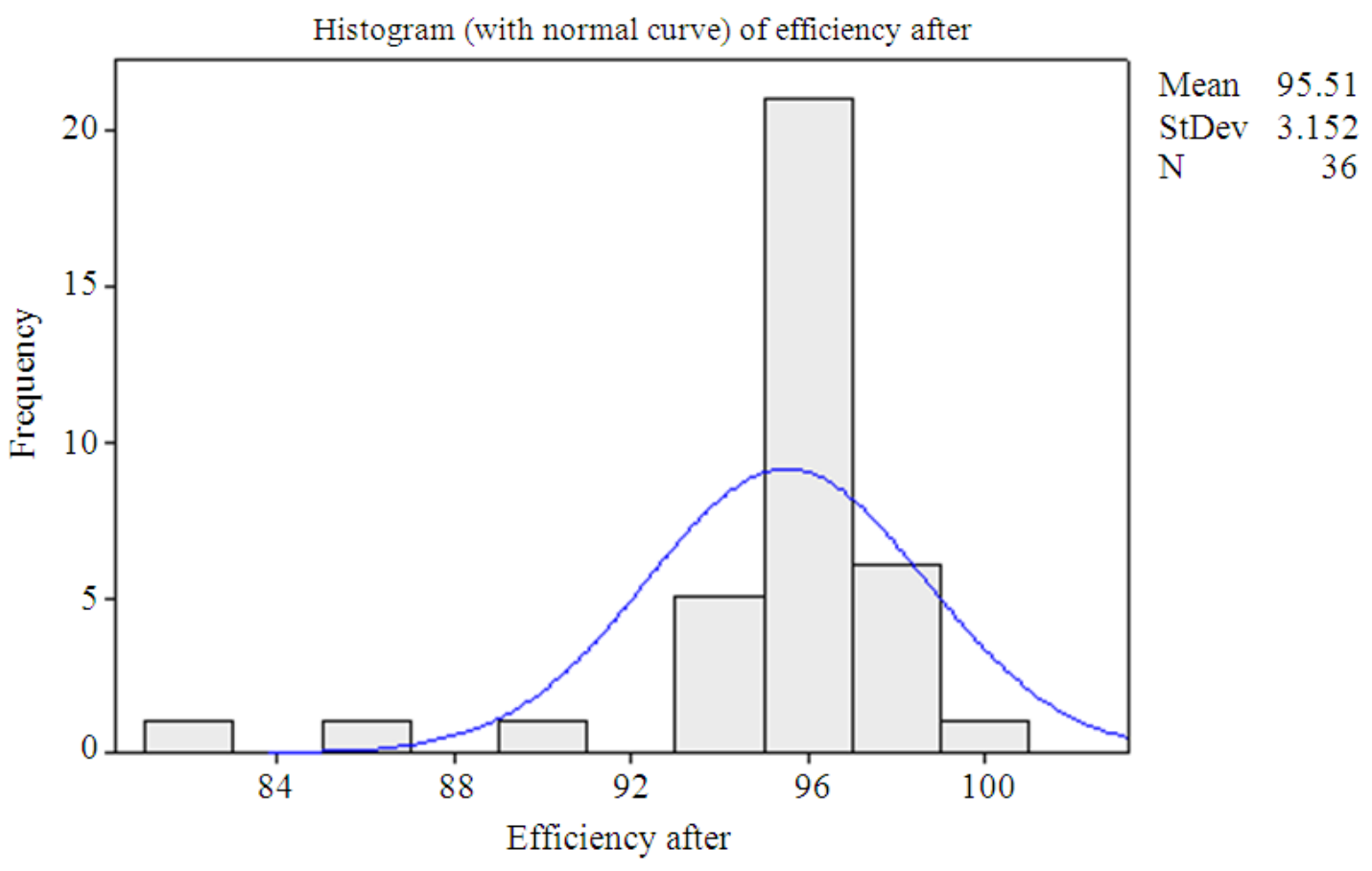

Fig. 9. Histogram of efficiency after considering the new scheduling method 


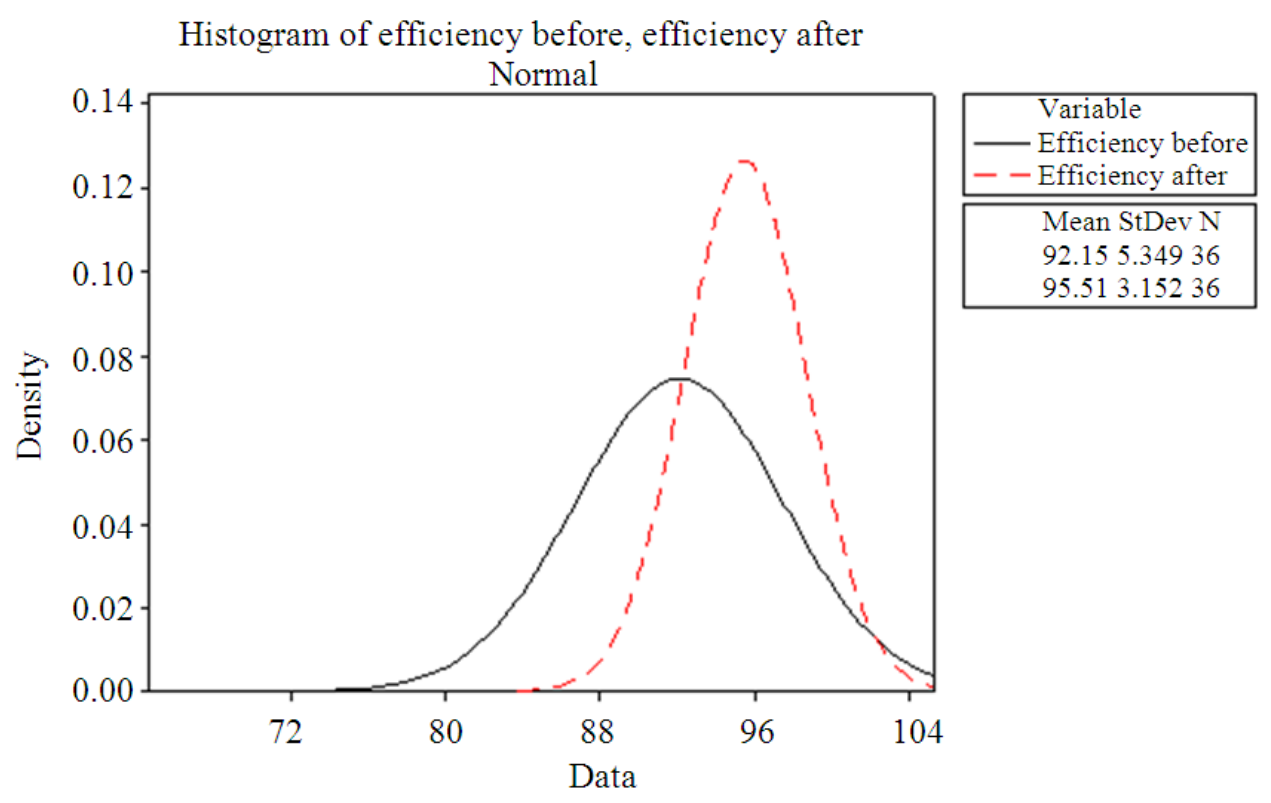

Fig. 10. Comparison efficiency of paint shop of IKCO before and after considering new method of scheduling

Table 1. Descriptive statistics: Efficiency of system before and after new scheduling method

\begin{tabular}{lllrrrr}
\hline Variable & Number of samples & Mean & StDev & Variance & Minimum & Maximum \\
\hline Efficiency before & 36 & 92.147 & 5.347 & 28.608 & 96.231 & 96.154 \\
Efficiency after & 36 & 95.513 & 3.125 & 9.932 & 82.892 & 100.000 \\
\hline
\end{tabular}

\section{CONCLUSION}

As mentioned the first objective was obtained by using MATLAB Distributed Computing Server (DCS) software. The methodology used in this study is the definition of job scheduler and positioning jobs in temporary queue and sorting via developing bubble sort in MATLAB distributed computing software. This is conducted on the basis of three factors of priority, time action and time stamp.

The second objective was achieved via Cause and Effect Analysis method (CAEA). In this regard, the failures in the miscellany of groups including material, equipment, people, maintenance, management and environment have been categorize. Accordingly, based on the simulated scheduling algorithm, the faults of ignoring the priority and time action of jobs are spontaneously eliminated from the system. Minitab software is used to show the status of the system before and after applying the proposed algorithm.

In a nutshell, the results of applying the suggested algorithm lead to the significant increase in system efficiency. It is suggested for future research to formulate some algorithms that will prevent job starvation in the industrial manufacturing environments.

\section{ACKNOLEDGMENT}

We thank The University of Toledo and The University of Putra Malaysia for providing fund. The International Car Manufacturing of Iran Khodro (http://www.ikco.com).

\section{REFERENCES}

Ahmad, I. and M.F. Othman, 2010. A backward recovery mechanism in preemptive utility accrual real time scheduling algorithm. J. Comput. Sci., 6: 728-734. DOI: 10.3844 jessp. 2010.728.734

AL-Hinai, N. and T. Elmekkawy, 2011. An efficient hybridized genetic algorithm architecture for the flexible job shop scheduling problem. Flexible Services Manufactur. J., 23: 64-85. DOI: 10.1007/s10696-010-9067-y 
Baker, K.R., 1974. Scheduling a full-time workforce to meet cyclic staffing requirements. Manage. Sci., 20: 1561-1568. DOI: $10.1287 / \mathrm{mnsc} .20 .12 .1561$

Chen, J.K., 2007. Utility priority number evaluation for FMEA. J. Failure Anal. Prevent., 7: 321-328. DOI: 10.1007/s1 1668-007-9060-2

Chen, J.S., 2010. Integration of job scheduling with delivery vehicle routing. Inform. Technol. J., 9: 1202-1206. DOI: $10.3923 /$ itj.2010.1202.1206

Emami-Mehrgani, B., S. Nadeau and J.P. Kenne, 2011. Lockout/tagout and operational risks in the production control of manufacturing systems with passive redundancy. Int. J. Product. Econ., 132: 165-173. DOI: 10.1016/j.ijpe.2011.03.028

Gao, H. and X. Liu, 2007. Improved artificial immune algorithm and its application on the permutation flow shop sequencing problems. Inform. Technol. J., 6: 929933. DOI: 10.3923/itj.2007.929.933

Ghoul, R.H., A. Benjelloul, S. Kechida and H. Tebbikh, 2007. A scheduling algorithm based on petri nets and simulated annealing. Am. J. Applied Sci., 4: 269-273. DOI: 10.3844/ajassp.2007.269.273

Hassan, M.K., A. Kandeil and A. Elkhayat, 2011. Improving oil refinery productivity through enhanced crude blending using linear programming modeling. Asian J. Sci. Res., 4: 95-113. DOI: 10.3923/ajsr.2011.95.113

Muzaffer, K. and M. Alikalfa, 2011. Learning IF-THEN priority rules for dynamic job shops using genetic algorithms. Robotics Comput. Integrated Manufactur., 27: 47-55. DOI: 10.1016/j.rcim.2010.06.001

Luqiao, F., 2010. An improved job shop scheduling algorithm based on efficiency function. Proceedings of the 2nd International Conference on Information Engineering and Computer Science, Dec. 25-26, IEEE Xplore Press, Wuhan, pp: 1-5. DOI: 10.1109/ICIECS.2010.5678327

Mahdavi, L. and B. Shirazi. 2010. A review of simulationbased intelligent decision support system architecture for the adaptive control of flexible manufacturing systems. J. Artificial Intelligence, 3: 201-219. DOI: 10.3923/jai.2010.201.219

Nojabaei, S., Z. Leman, S.H. Tang and S. Sulaiman, 2012. Development of priority oriented scheduling method to increase the reliability of manufacturing systems. Am. J Applied Sci., 9: 1435-1442. DOI: 10.3844/ajassp.2012.1435.1442

Omar, M.K., Y.A.P. Suppiah and S.C. Teo, 2005. Development of integrated production scheduling system in the process industry. J. Comput. Sci., 1: 395-399. DOI: $10.3844 /$ jessp.2005.395.399
Omar, M.S., 2007. On the solution of the problem of scheduling unrelated parallel machines with machine eligibility restrictions under fuzziness. J. Applied Sci. Res., 2: 404-411.

Papazachos, Z.C. and H.D. Karatza, 2010. Performance evaluation of bag of gangs scheduling in a heterogeneous distributed system. J. Syst. Software, 83: 1346-1354. DOI: 10.1016/j.jss.2010.01.009

Pinedo, M.L., 2012. Scheduling: Theory, algorithms and systems. 1st Edn., Springer Science and Business Media, New York, ISBN-10: 1461423619, pp: 693.

Qiming, T., L. Li, J. Ling and B. Xinxin, 2009. A novel dynamic priority scheduling algorithm of process engine in SOA. Proceedings of the IEEE International Conference on Web Services, Jul. 610, IEEE Xplore Press, Los Angeles, CA, pp: 711-718. DOI: 10.1109/ICWS.2009.17

Rashid, M.M. and N.M. Akhtar, 2006. A new multilevel CPU scheduling algorithm. J. Applied Sci., 6: 2036-2039. DOI: 10.3923/jas.2006.2036.2039

Samreen, F. and M.S.H. Khiyal, 2007. Q-learning scheduler and load balance for heterogeneous systems. J. Applied Sci., 7: 1504-151. DOI: 10.3923/jas.2007.1504.1510

Taghavifard, M.T., M. Heydar and S.S. Mousavi, 2009. A genetic algorithm for scheduling flexible manufacturing cells. J. Applied Sci., 9: 97-104. DOI: $10.3923 /$ jas.2009.97.104

Taiwo, A.S., 2007. Determination of the optimal manpower size using linear programming model. Res. J. Bus. Manage., 1: 30-36. DOI: 10.3923/rjbm.2007.30.36

Tzimopoulos, C., L. Mpallas and C. Evangelides, 2008. Fuzzy model comparison to extrapolate rainfall data. J. Environ. Sci. Technol., 1: 214224. DOI: 10.3923 /jest.2008.214.224

Venkatachalam, S, C. Arumugum, K. Raja and V. Selladurai, 2008. Quality function deployment in agile parallel machine scheduling through neural network scheduling. Asian J. Sci. Res., 1: 146-152. DOI: 10.3923/ajsr.2008.146.152

Zang, R., J. Bai, H. Xu, L. Zhang and J. Wu et al., 2011. Selection of suitable reference genes for real-time quantitative PCR studies in lanzhou fat-tailed sheep (Ovis aries). Asian J. Sci. Res., 6: 789-804. 